\title{
Regularity and geometric properties of solutions of the Einstein-Vacuum equations
}

\author{
Sergiu Klainerman Igor Rodnianski
}

\begin{abstract}
We review recent results, obtained in [Kl-Ro1]-[Kl-Ro3], concerning the study of rough solutions to the initial value problem for the Einstein vacuum equations expressed relative to wave coordinates. We develop new analytic methods based on Strichartz type inequalities which results in a gain of half a derivative relative to the classical result. Our methods blend paradifferential techniques with a geometric approach to the derivation of decay estimates. The latter allows us to take full advantage of the specific structure of the Einstein equations.
\end{abstract}

\section{Introduction}

This paper is concerned with the issue of low regularity (rough) solutions of the Einstein Vacuum equations

$$
\mathbf{R}_{\alpha \beta}(\mathbf{g})=0, \quad \alpha, \beta=0, . ., 3
$$

where $\mathbf{g}$ is an unknown four dimensional Lorentz metric and $\mathbf{R}_{\alpha \beta}$ is its Ricci curvature tensor.

We prescribe the initial data, the Riemannian three dimensional metric $g_{i j}$ and a symmetric tensor $k_{i j}$ on the space-like hypersurface $\Sigma_{0}=t=0$. The Cauchy problem for the equation (1) then consists of finding a metric $\mathbf{g}$ satisfying (1) with a property that the metric induced by $\mathbf{g}$ on $\Sigma_{0}$ coincides with the Riemannian metric $g$ and that the 2-tensor $k$ is the second fundamental form of the hypersurface $\Sigma_{0}$. The latter property can be expressed as follows. Let $T$ denote the unit vectorfield normal to the the level hypersurfaces of the time foliation $\Sigma_{t}$. Then $k_{i j}=-\left.\frac{1}{2} \mathcal{L}_{T} \mathbf{g}_{i j}\right|_{\Sigma_{0}}$, where $\mathcal{L}_{T}$ denotes the Lie derivative in the direction of the vectorfield $T$. 
It is well known that the Einstein equations form an overdetermined system. As a consequence, the initial data set $(g, k)$ cannot be prescribed arbitrarily and, in fact, satisfy the system of constraint equations

$$
\begin{aligned}
& \nabla^{j} k_{i j}-\nabla_{i} k_{j}^{j}=0, \\
& R-k_{i j} k^{i j}+k_{j}^{j} k_{i}^{i}=0,
\end{aligned}
$$

where the covariant derivative $\nabla$ is defined with respect to the metric $g$, and $R$ is the scalar curvature of $g$.

In a given system of local coordinates $x^{\alpha}, \alpha=0, . ., 3$ with $t=x^{0}$, the equation (1) can be written in the form

$$
\mathbf{g}^{\alpha \beta}\left(\partial_{\alpha \mu}^{2} \mathbf{g}_{\beta \nu}+\partial_{\beta \nu}^{2} \mathbf{g}_{\alpha \mu}-\partial_{\mu \nu}^{2} \mathbf{g}_{\alpha \beta}-\partial_{\alpha \beta}^{2} \mathbf{g}_{\mu \nu}\right)=N_{\mu \nu}(g, \partial g)
$$

which does not define an equation of a specific type (hyperbolic, elliptic,...). On the other hand, any solution of the Cauchy problem corresponding to the equation (1) can be unique only up to a diffeomorphism which preserves the induced metric $g$ and the second fundamental form $k$ on $\Sigma_{0}$. This is often referred to as the gauge freedom of the Einstein equations. The choice of a special gauge resolves the above ambiguity. It can also help in converting the Einstein equations into a more tractable form.

The focus of this work is the study of the Einstein Vacuum equations in the gauge described by the choice of the special wave coordinates $x^{\alpha}, \alpha=0, . ., 3$ which are set to satisfy the wave equation relative to the background metric $\mathbf{g}$.

$$
\square_{\mathbf{g}} x^{\alpha}=\frac{1}{\sqrt{|\mathbf{g}|}} \partial_{\mu}\left(\mathbf{g}^{\mu \nu} \sqrt{|\mathbf{g}|} \partial_{\nu}\right) x^{\alpha}=0
$$

In this coordinates, the Einstein vacuum equations take the reduced form, see [Br], $[\mathrm{H}-\mathrm{K}-\mathrm{M}]$.

$$
\mathbf{g}^{\alpha \beta} \partial_{\alpha} \partial_{\beta} \mathbf{g}_{\mu \nu}=N_{\mu \nu}(\mathbf{g}, \partial \mathbf{g}), \quad \mu, \nu=0, . ., 3
$$

with $N$ quadratic in the first derivatives $\partial \mathbf{g}$ of the metric. The reduced Einstein Vacuum equations (3) form a system of quasilinear hyperbolic equations.

Among other possible choices of gauge conditions we note the so called CMCSH (constant mean curvature + spatial harmonic coordinates) gauge given by the condition that the trace of the second fundamental form $k, k_{i}^{i}=$ const on each time slice $\Sigma_{t}=\{(t, x): t=$ const $\}$, together with the choice of harmonic coordinates $x^{i}$, $i=1,2,3$ satisfying the equations $\triangle_{g} x^{\alpha}=0$. Here we have extended the notations $g$ and $k$ to denote the induced Riemannian metric $g$ and second fundamental form $k$ on any level hypersurface $\Sigma_{t}$. It can be shown that the Einstein equations in this gauge are reduced to an elliptic-hyperbolic system for which the classical results on the Cauchy problem proved in the wave coordinate gauge (see Theorem 1.1) also hold [An-Mo].

We consider the initial value problem for the equation (3) along the space-like hyperplane $\Sigma_{0}$ given by $t=x^{0}=0$,

$$
\nabla \mathbf{g}_{\alpha \beta}(0) \in H^{s-1}\left(\Sigma_{0}\right), \quad \partial_{t} \mathbf{g}_{\alpha \beta}(0) \in H^{s-1}\left(\Sigma_{0}\right)
$$


with $\nabla$ denoting the gradient with respect to the space coordinates $x^{i}, i=1,2,3$ and $H^{s}$ the standard Sobolev spaces. We also assume that $\mathbf{g}_{\alpha \beta}(0)$ is a continuous Lorentz metric and $\sup _{|x|=r}\left|\mathbf{g}_{\alpha \beta}(0)-\mathbf{m}_{\alpha \beta}\right| \longrightarrow 0$ as $r \longrightarrow \infty$, where $|x|=\left(\sum_{i=1}^{3}\left|x^{i}\right|^{2}\right)^{\frac{1}{2}}$ and $\mathbf{m}_{\alpha \beta}$ the Minkowski metric.

The following local existence and uniqueness result (well posedness) is well known (see $[\mathrm{H}-\mathrm{K}-\mathrm{M}]$ and the previous result of $\mathrm{Ch}$. Bruhat $[\mathrm{Br}]$ for $s \geq 4$.)

Theorem 1.1. Consider the reduced equation (3) subject to the initial conditions (4) for some $s>5 / 2$. Then there exists a time interval $[0, T]$ and unique (Lorentz metric) solution $\mathbf{g} \in C^{0}\left([0, T] \times \mathbb{R}^{3}\right), \partial \mathbf{g}_{\mu \nu} \in C^{0}\left([0, T] ; H^{s-1}\right)$ with $T$ depending only on the size of the norm $\left\|\partial \mathbf{g}_{\mu \nu}(0)\right\|_{H^{s-1}}$.

We establish a significant improvement of this result bearing on the issue of minimal regularity of the initial conditions:

Main Theorem. Consider a classical solution of the equations (3) for which (1) also holds ${ }^{1}$. We show that the time $T$ of existence depends in fact only on the size of the norm $\|\partial \mathbf{g}(0)\|_{H^{s-1}}$, for any fixed $s>2$. The constructed solution satisfies the Strichartz estimate

$$
\|\partial \mathbf{g}\|_{L_{t}^{2} L_{x}^{\infty}} \leq C\|\partial \mathbf{g}(0)\|_{H^{s-1}}
$$

Remark 1.2. Theorem 1.1 implies the classical local existence result of $[\mathrm{H}-\mathrm{K}-\mathrm{M}]$ for asymptotically flat initial data sets $\Sigma, g, k$ with $\nabla g, k \in H^{s-1}(\Sigma)$ and $s>\frac{5}{2}$. Uniqueness can be proved for additional regularity $s>1+\frac{5}{2}$.

Remark 1.3. The Main Theorem ought to imply existence and uniqueness ${ }^{2}$ for initial conditions with $H^{s}, s>2$, regularity. To achieve this we only need to approximate a given $H^{s}$ initial data set ( i.e. $\nabla g \in H^{s-1}\left(\Sigma_{0}\right), k \in H^{s-1}\left(\Sigma_{0}\right), s>2$ ) for the Einstein vacuum equations by classical initial data sets, i.e. $H^{s^{\prime}}$ data sets with $s^{\prime}>\frac{5}{2}$, for which theorem 1.1 holds. The Main Theorem allows us to pass to the limit and derive existence of solutions for the given, rough, initial data set. We don't know however if such an approximation result for the constraint equations exists in the literature.

One of the aspects of our result is a new connection between the propagation of regularity for the solutions of the Einstein equations addressed in the Main Theorem and the geometry induced by the above metric solutions. Here by the geometry we mean the generated local causal structure [Ha-El]. In comparison, the geometry associated with a classical solution of the Einstein equations is irrelevant to the proof of the classical result. It in fact only uses the Lorentzian character of the uknown metric $\mathbf{g}$.

To explain the above connection we now describe the relevant geometric concepts and state the geometric counterpart of the Main Theorem. In Minkowski space one can define two special global foliations $C_{u}$ and $S_{t, u}$. The foliation $C_{u}$ is that of the

\footnotetext{
${ }^{1}$ In other words for any solution of the reduced equations (3) whose initial data satisfy the constraint equations, see $[\mathrm{Br}]$ or $[\mathrm{H}-\mathrm{K}-\mathrm{M}]$. The fact that our solutions verify (1) plays a fundamental role in our analysis.

${ }^{2}$ Properly speaking uniqueness holds, with $s>2$, only for the reduced equations. Uniqueness for the actual Einstein equations requires one more derivative, see [H-K-M].
} 
light cones $C_{u}=\{(t, x): t-|x|=u\}$, dependent on the parameter $u$. This is also the foliation generated by the level surfaces of the optical function $u(t, x):=t-|x|$. The foliation $S_{t, u}$ of the 2-dimensional spheres, dependent on two parameters $t$ and $u$, is obtained by intersecting the light cones $C_{u}$ with the time slices $t=$ const. The optical function $u$ is a solution of the eikonal equation

$$
m^{\alpha \beta} \partial_{\alpha} u \partial_{\beta} u=0
$$

associated with the Minkowski metric $m$. The light cones $C_{u}$ are generated by the vectorfield

$$
L=-m^{\alpha \beta} \partial_{\alpha} u \partial_{\beta}=\partial_{t}+\partial_{r}
$$

which is null, i.e., $m(L, L)=0$. One cal also define the complementary null vectrofield $\underline{L}=\partial_{t}-\partial_{r}$ so that the vectorfields $L, \underline{L}$ form a so called null pair. Finally, adding two orthonormal vectorfields $e_{A}, A=1,2$ tangent to the 2 -spheres $S_{t, u}$ defines a null frame $\left(L, \underline{L}, e_{A}\right)$.

Let now $\mathrm{g}$ be an arbitrary smooth Lorentzian metric of the form

$$
\mathbf{g}=-\mathbf{n}^{2} d t^{2}+g_{i j}\left(d x^{i}+\mathbf{v}^{i} d t\right)\left(d x^{j}+v^{j} d t\right)
$$

where $g_{i j}$ is a Riemannian metric on slices $\Sigma_{t}, \mathbf{n}$ is a lapse function, and $\mathbf{v}$ is a vector-valued shift function. We can define the associated light or null cones $C_{u}$ by means of the optical function $u$ which is now a solution of the corresponding eikonal equation

$$
\mathbf{g}^{\alpha \beta} \partial_{\alpha} u \partial_{\beta} u=0
$$

with the initial data $\left.u\right|_{\Gamma_{t}}=t$, where $\Gamma_{t}$ is the analogue of the time axis defined to be a time-like geodesic orthogonal to the time slices $\Sigma_{t}$. It is not too difficult to see that for a general metric $\mathbf{g}$ the eikonal equation (6) admits only a local solution. As a result it is only a small neighborhood of the time axis $\Gamma_{t}$ that can be shown to be foliated by the light cones $C_{u}$ and the corresponding 2-surfaces $S_{t, u}$. The latter are again obtained by intersecting $C_{u}$ with the time slices $\Sigma_{t}$. Similarly, we define the null pair $(L, \underline{L})$ with

$$
L=-\mathbf{b g}^{\alpha \beta} \partial_{\alpha} u \partial_{\beta}, \quad \underline{L}=2 T-L
$$

and $\mathbf{b}^{-1}=T(u)$ is the null lapse function measuring the separation between the null cones $C_{u}$. We can complement $(L, \underline{L})$ by a pair of orthonormal vectorfields $e_{A}$ tangent to the 2 -surfaces $S_{t, u}$ to define a null frame $\left(L, \underline{L}, e_{A}\right)$.

Let $\langle$,$\rangle denote the scalar product with respect to the metric \mathbf{g}, D$ be a covariant derivative associated with $\mathbf{g}$, let $\nabla$ stand for the covariant derivative induced on $\Sigma_{t}$, and $\not$ be the corresponding covariant derivative on $\S_{t, u}$.

The foliations $C_{u}$ and $S_{t, u}$ can be shown to be quantitatively controlled by means of the following geometric quantities.

$$
\begin{aligned}
& \chi_{A B}=\left\langle D_{e_{A}} L, e_{B}\right\rangle, \quad \eta_{A}=\frac{1}{2}\left\langle D_{\underline{L}} L, e_{A}\right\rangle, \\
& k_{i j}=-\frac{1}{2} \mathcal{L}_{T} g_{i j},
\end{aligned}
$$


where $\chi_{A B}$ is the null second fundamental form of the 2-surfaces $S_{t, u}, \eta_{A}$ is the so called torsion, and $k_{i j}$ is the second fundamental form of the level surfaces $\Sigma_{t}$. The quantities $\chi$ and $\eta$ are null components of the Hessian $D^{2} u$ of the optical function. The second fundamental form $\chi_{A B}$ can be further decomposed into its trace and traceless parts

$$
\chi_{A B}=\frac{1}{2} \operatorname{tr} \chi \delta_{A B}+\hat{\chi}_{A B}
$$

One of the basic and most important properties of the $S_{t, u}$ foliation is the area growth. In Minkowski space, $S_{t, u}$ is the sphere of radius $t-u$ and thus $A\left(S_{t, u}\right)=$ $4 \pi(t-u)^{2}$ grows quadratically with the increase of the parameter $r=t-u$. In the situation of a general smooth Lorentzian metric $\mathbf{g}$ this property also holds in the sense that $A\left(S_{t, u}\right) \approx(t-u)^{2}$ at least in a small neighborhood of the time axis. The area growth is in fact related to the behavior of $\operatorname{tr} \chi$, which in Minkowski space is precisely $\frac{2}{t-u}$ and in the general smooth case can be shown to be close to the quantity $\frac{2}{n(t-u)}$.

Our interest however is in the geometric properties of the rough Lorentzian metrics $\mathbf{g}$. In particular, we are interested in metrics $\mathbf{g}$ with the regularity comparable to the one described in the Main Theorem. It is easy to see that for a general Lorentzian metric $\mathbf{g}$ with the property that $\partial \mathbf{g}(t, \cdot)$ only belongs to $H^{s-1}$ for some $s>2$ sufficiently close to $s=2$ and with a finite Strichartz norm $\|\partial \mathbf{g}\|_{L_{t}^{2} L_{x}^{\infty}}$, even the local picture does not survive. The light cones $C_{u}$, for example, may collapse instantaneously. The situation changes however in the case of the Einstein metrics. We have the following companion of the Main Theorem.

Geometric Theorem. Consider a classical solution of the equations (3) for which (1) also holds. Then any sufficiently small neighborhood of space-time can be foliated by the family of light cones ${ }^{3} C_{u}$ or the 2-surfaces $S_{t, u}$ generated by the optical function $u$ defined in (6). The size of a neighborhood depends only on the size of the norm $\|\mathbf{\|}\| \|_{s}$ defined as follows

$$
|\|\mathbf{g}\||_{s}:=\|\partial \mathbf{g}\|_{L_{t}^{\infty} H^{s-1}}+\|\partial g\|_{L_{t}^{2} L_{x}^{\infty}}+\left\|D_{*} g\right\|_{L_{u}^{\infty} H^{s-1}\left(C_{u}\right)}
$$

where $D_{*}$ denotes any tangential to the light cone $C_{u}$ derivative and $H^{s-1}\left(C_{u}\right)$ refers to the restriction of the standard $H^{s-1}\left(\mathbf{R}^{4}\right)$ Sobolev space to the light cone $C_{u}$. Moreover, in that region the following estimates on the geometric quantities $\chi, \eta$, and $k$ associated with our foliations hold for any fixed value of the parameter $s>2$.

$$
\begin{aligned}
& \left\|\operatorname{tr} \chi-\frac{2}{n(t-u)}\right\|_{L_{t}^{2} L_{x}^{\infty}}+\|\hat{\chi}\|_{L_{t}^{2} L_{x}^{\infty}}+\|\eta\|_{L_{t}^{2} L_{x}^{\infty}}+\|k\|_{L_{t}^{2} L_{x}^{\infty}} \leq C\left(\|\| \mathbf{g} \|\left.\right|_{s}\right), \\
& \left\|\sup _{u \geq \frac{t}{2}}\right\| D\left(\operatorname{tr} \chi-\frac{2}{n(t-u)}\right)\left\|_{L^{2}\left(S_{t, u}\right)}\right\|_{L_{t}^{1}} \leq C\left(\|\| \mathbf{g}\|\|_{s}\right)
\end{aligned}
$$

These estimates in particular imply the growth property of $S_{t, u}, A\left(S_{t, u}\right) \approx(t-u)^{2}$.

\footnotetext{
${ }^{3}$ with vertices an a time-line geodesic $\Gamma_{t}$ orthogonal to $\Sigma_{t}$ and passing through the above neighborhood
} 
Remark 1.4. Our Main Theorem implies that the first two quantities in the definition of the \|\| $\mathbf{g} \|\left.\right|_{s}$ can be controlled purely in terms of $\|\partial g(0)\|_{H^{s-1}}$ norm of the initial data provided that $s>2$ and the above space-time neighborhood intersects the initial hypersurface $\Sigma_{0}$. In fact, the proof of the Main Theorem also gives control of the quantity $\left\|D_{*} g\right\|_{L_{u}^{\infty} H^{s-1}\left(C_{u}\right)}$. This is achieved by proving the analogue of the characteristic energy estimate for solutions of the linear wave equation. Therefore, combination of the Main and Geometric theorems gives a stronger result in which the size of a neighborhood depends only on the norm $\|\partial g\|_{H^{s-1}\left(\Sigma_{t}\right)}$ for some value of $s>2$ and a time slice $\Sigma_{t}$ intersecting the above neighborhood. Perhaps more interesting is the fact that the proof of the Main Theorem itself crucially relies on the information available from the Geometric Theorem.

Remark 1.5. In [Kl-Ro2] we prove a slightly weaker version of the Geometric Theorem. More precisely, we define the frequency truncated metric

$$
\mathbf{g}_{<\lambda}=P_{<\lambda} \mathbf{g}
$$

where $P_{<\lambda}$ is the Littlewood-Paley projector on the frequencies $<\lambda$. This projector is a multiplier in Fourier space represented by a function $\zeta\left(\lambda^{-1} \xi\right)$ for some smooth compactly supported function $\zeta$ equal to one on the ball of radius one. We then show that for all sufficiently large $\lambda$ and for any $\epsilon>0$ the foliations $C_{u}$ and $S_{t, u}$ defined relative to the metric $\mathbf{g}_{<\lambda}$ exist and satisfy estimates (10) and (11) on any neighborhood of the size $\lambda^{-\epsilon} C\left(\|\mid g\|_{H^{s}}\right)$. This is an " $\epsilon$ - weaker" result than the Geometric Theorem. However, we believe that the stronger conclusion of the Geometric Theorem also holds.

\section{Main ideas of the Proof}

For convenience we shall also write the reduced equations (3) in the form of a system of quasilinear wave equations of the form,

$$
\mathbf{g}^{\alpha \beta} \partial_{\alpha} \partial_{\beta} \phi=N(\phi, \partial \phi)
$$

where $\phi=\left(\mathbf{g}_{\mu \nu}\right), N=N_{\mu \nu}$ and $\mathbf{g}^{\alpha \beta}=\mathbf{g}^{\alpha \beta}(\phi)$. The classical local existence result for systems of wave equations of type (12) is based on energy estimates and the standard $H^{s} \subset L^{\infty}$ Sobolev inequality. Indeed using energy estimates and simple commutation inequalities one can show that,

$$
\|\partial \phi(t)\|_{H^{s-1}} \leq E\|\partial \phi(0)\|_{H^{s-1}}
$$

with $E=\exp \left(C \int_{0}^{t}\|\partial \phi(\tau)\|_{L_{x}^{\infty}} d \tau\right)$. By the classical Sobolev inequality,

$$
E \leq \exp \left(C t \sup _{0 \leq \tau \leq t}\|\partial \phi(\tau)\|_{H^{s-1}} d \tau\right)
$$

provided that $s>\frac{5}{2}$. The classical local existence result follows by combining this last estimate, for a small time interval, with the energy estimates (13). This scheme 
is very wasteful. To do better one would like to take advantage of the mixed $L_{t}^{1} L_{x}^{\infty}$ norm appearing in the definition of $E$. If $\phi$ is a solution of the standard wave equation

$$
\square \phi=0
$$

in Minkowski space, we have the following Strichartz type inequality:

$$
\|\partial \phi\|_{L_{t}^{2} L_{x}^{\infty}\left([0, T] \times \mathbb{R}^{3}\right)} \leq C T^{\epsilon}\|\partial \phi(0)\|_{H^{1+\epsilon}} .
$$

with $\epsilon>0$ arbitrarily small. Based on this fact it was reasonable to hope that one can improve the Sobolev exponent in the classical local existence theorem from $s>\frac{5}{2}$ to $s>2$. This can be easily done for solutions of semilinear wave equations, see $[\mathrm{Po}-\mathrm{Si}]$. In the case of general quasilinear wave equations of the form (12), however, the situation is far more difficult. One can no longer rely on the Strichartz inequality (15) for the flat D'Alembertian in (14); we need instead its extension to the operator $\mathbf{g}^{\alpha \beta} \partial_{\alpha} \partial_{\beta}$ appearing in (12). Moreover, since the metric $\mathbf{g}^{\alpha \beta}$ depends on the solution $\phi$, it can have only as much regularity as $\phi$ itself. This means that we have to confront the issue of proving Strichartz estimates for wave operators $\mathbf{g}^{\alpha \beta} \partial_{\alpha} \partial_{\beta}$ with very rough coefficients $\mathbf{g}^{\alpha \beta}$. This issue was recently addressed in the pioneering works of Smith [Sm], Bahouri-Chemin [Ba-Ch1], [Ba-Ch2] and Tataru [Ta1], [Ta2], we refer to the introduction in [Kl1] and [Kl-Ro] for a more thorough discussion of their important contributions. The results of Bahouri-Chemin and Tataru are based on establishing a Strichartz type inequality, with a loss, for wave operators with very rough coefficients ${ }^{4}$. The optimal result ${ }^{5}$ in this regard, due to Tataru, see [Ta2], requires a loss of $\sigma=\frac{1}{6}$. This leads to a proof of local well posedness for systems of type (12) with $s>2+\frac{1}{6}$.

To do better than that one needs to take into account the nonlinear structure of the equations. In [Kl-Ro] we were able to improve the result of Tataru by taking into account not only the expected regularity properties of the coefficients $\mathbf{g}^{\alpha \beta}$ in (12) but also the fact that they are themselves solutions to a similar system of equations. This allowed us to improve the exponent $s$, needed in the proof of well posedness of equations of type ${ }^{6}(12)$, to $s>2+\frac{2-\sqrt{3}}{2}$. Our approach was based on a combination of the paradifferential calculus ideas, initiated in [Ba-Ch1] and [Ta2], with a geometric treatment of the actual equations introduced in [K11]. The main improvement was due to a gain of conormal differentiability for solutions to the eikonal equations

$$
\mathbf{g}_{<\lambda}^{\alpha \beta} \partial_{\alpha} u \partial_{\beta} u=0
$$

That gain could be traced down to the fact that a certain component of the Ricci curvature of the metric $h=\mathbf{g}_{<\lambda}$ has a special form. More precisely recalling vectorfield $L$, the null generator of the light cones $C_{u}$ we found that the null Ricci

\footnotetext{
${ }^{4}$ The derivatives of the coefficients $\mathbf{g}$ are required to be bounded in $L_{t}^{\infty} H_{x}^{s-1}$ and $L_{t}^{2} L_{x}^{\infty}$ norms, with $s$ compatible with the regularity required on the right hand side of the Strichartz inequality one wants to prove.

${ }^{5}$ Recently Smith-Tataru [Sm-Ta1] have shown that the result of Tataru is indeed sharp.

${ }^{6}$ The result in [Kl-Ro] applies to general equations of type (12) not necessarily tied to (1). In [Kl-Ro] we have also made the simplifying assumptions $\mathbf{n}=1$ and $\mathbf{v}=0$.
} 
component $\mathbf{R}_{L L}=\mathbf{R i c}(h)(L, L)$, verifies the remarkable identity:

$$
\mathbf{R}_{L L}=L(z)-\frac{1}{2} L^{\mu} L^{\nu}\left(h^{\alpha \beta} \partial_{\alpha} \partial_{\beta} h_{\mu \nu}\right)+\mathrm{e}
$$

where $z \leq O(|\partial h|)$ and $e \leq O\left(|\partial h|^{2}\right)$. Thus, apart from $L(z)$ which is to be integrated along the null geodesic flow generated by $L$, the only terms which depend of the second derivatives of $h$ appear in $h^{\alpha \beta} \partial_{\alpha} \partial_{\beta} h$ and can therefore be eliminated with the help of the equations (12).

In [Kl-Ro1]-[Kl-Ro3] we developed the ideas of [Kl-Ro] further by taking full advantage of the the structure of the Einstein equations (1). An important aspect of our analysis is that the term $L(z)$ appearing on the right hand side of (17) vanishes identically. We make use of both the vanishing of the Ricci curvature of $\mathbf{g}$ and the wave coordinate condition (2). The other important new features are the use of energy estimates along the null hypersurfaces generated by the optical function $u$ and a deeper use of the conormal properties of the null structure equations.

In [Sm-Ta2] H. Smith and D. Tataru obtained the parallel $H^{s}, s>2$ local well posedness result for general quasilinear equations, as well as the new improved results in other dimensions rather than $n=3$. For general equations their results in dimensions two and three are sharp due to the counterexamples of H. Lindblad [Li]. Their approach is based on the construction of a wave packet approximation of a solution. The geometry of wave packets controls the desired Strichartz estimate. The construction relies on the foliation by the null planes. It uses a gain of differentiablity along each plane, which can be traced to the decomposition of the tangential components of the curvature in the spirit (17), but avoids references to the regularity of the foliation in the direction transversal to the leafs (i.e. torsion of the foliation).

We strongly believe that the result of our main theorem is not sharp. The critical Sobolev exponent for the Einstein equations is $s_{c}=\frac{3}{2}$. A proof of well posedness for $s=s_{c}$ will provide a much stronger version of the global stability of Minkowski space than that of [Ch-Kl]. This is completely out of reach at the present time. A more reasonable goal is to prove the $L^{2}$ - curvature conjecture, see [K12], corresponding to the exponent $s=2$.

Below we outline the main steps in the proofs of the Main and Geometric theorems. The details are contained in [Kl-Ro1]-[Kl-Ro3].

\section{Bootstrap}

The proof of the Main Theorem is based on a bootstrap argument in which we assume that the inequality

$$
\|\partial \phi\|_{L_{[0, T]}^{\infty} H^{s-1}}+\|\partial \phi\|_{L_{[0, T]}^{2} L_{x}^{\infty}} \leq B_{0}
$$

holds for some positive constant $B_{0}$ on a time interval $[0, T]$. To achieve the conclusion of the Main Theorem we establish that the constants $B_{0}$ and $T$ depend, in fact, only on the size of the initial data $\|\partial \phi(0)\|_{H^{s-1}}$ for any fixed $s>2$. The energy estimate (13) implies that the desired conclusion would follow from the Strichartz 
estimate

$$
\|\partial \phi\|_{L_{[0, T]}^{2} L_{x}^{\infty}} \leq C\left(B_{0}\right) T^{\delta}
$$

for some $\delta>0$. This is the key estimate.

\section{Linearization}

Since the right hand-side of the equation (12) contains only the terms which depend only on $\phi$ and quadratically on $\partial \phi$ we can reduce the proof of (19) to the corresponding Strichartz estimate for a solution of the linear wave equation $\mathbf{g}^{\alpha \beta} \partial_{\alpha} \partial_{\beta} \psi=0$. This is done with the help of the Duhamel formula. The desired estimate now takes the form

$$
\|\partial \psi\|_{L_{[0, T]}^{2} L_{x}^{\infty}} \leq C\left(B_{0}\right) T^{\delta}\|\psi(0)\|_{H^{2+\gamma}}
$$

for some $0<\gamma<s-2$.

\section{Microlocalization}

We introduce the Littlewood-Paley projectors $P_{\lambda}$ truncating all the frequencies outside of the dyadic shell of size $\lambda$. It is easy to see that the estimate (20) can be replaced by a dyadic Strichartz estimate

$$
\left\|\partial P_{\lambda} \psi\right\|_{L_{[0, T]}^{2} L_{x}^{\infty}} \leq C\left(B_{0}\right) c_{\lambda} T^{\delta}\|\psi(0)\|_{H^{2+\gamma}}, \quad \sum_{\lambda} c_{\lambda} \leq 1
$$

for all sufficiently large values of the dyadic parameter $\lambda$. We can furthermore derive a wave equation for the function $P_{\lambda} \psi$. Using the standard techniques of the Bony's paradifferential calculus [Ba-Ch1], [Ta2], [K11], [Kl-Ro1] and the Duhamel formula we can make a reduction to the Strichartz estimate

$$
\left\|P_{\lambda} \partial \psi\right\|_{L_{I}^{2} L_{x}^{\infty}} \leq C\left(B_{0}\right) \bar{T}^{\delta}\|\partial \psi(0)\|_{\dot{H}^{1+\delta}}
$$

where $\psi$ now is a solution of $\mathbf{g}_{<\lambda}^{\alpha \beta} \partial_{\alpha} \partial_{\beta} \psi=0$, with initial conditions at $t=0$ verifying, $\left(2^{-10} \lambda\right)^{m} \leq\left\|\nabla^{m} \partial \psi(0)\right\|_{L_{x}^{2}} \leq\left(2^{10} \lambda\right)^{m}\|\partial \psi(0)\|_{L_{x}^{2}}$. The time interval $I$ which can be assumed to be the interval $[0, \bar{T}]$ is now restricted to the size $\leq \lambda^{-\epsilon}$ for some $\epsilon>0$.

\section{Rescaling}

Introduce the rescaled metric $H(t, x)=\mathbf{g}_{<\lambda}\left(\lambda^{-1} t, \lambda^{-1} x\right)$ and consider the rescaled equation $H^{\alpha \beta} \partial_{\alpha} \partial_{\beta} \psi=0$ in the region $\left[0, t_{*}\right] \times \mathbb{R}^{3}$ with $t_{*} \leq \lambda^{1-\epsilon}$. Then, with $P=P_{1}$,

$$
\|P \partial \psi\|_{L_{I}^{2} L_{x}^{\infty}} \leq C\left(B_{0}\right) t_{*}^{\delta}\|\partial \psi(0)\|_{L^{2}}
$$

would imply the estimate (21). 


\section{Reduction to a localized $L^{2}-L^{\infty}$ decay estimate}

The standard way to prove a Strichartz inequality of the type discussed above is to reduce it, by a $T T^{*}$ type argument, to an $L^{1}-L^{\infty}$ dispersive type inequality. However an additional spatial localization allows us to replace it with the $L^{2}-L^{\infty}$ decay estimate. We state this as the following theorem (see [Kl-Ro1]):

Theorem 2.1. Let $\psi$ be a solution of the equation, $\square_{H} \psi=0$ on the time interval $\left[0, t_{*}\right]$ with $t_{*} \leq \lambda^{1-\epsilon}$ Assume that the initial data is given at $t=t_{0} \in\left[0, t_{*}\right]$, supported in the ball $B_{\frac{1}{2}}(0)$ of radius $\frac{1}{2}$ centered at the origin. There exists a function $d(t)$, with $t_{*}^{1 / q}\|d\|_{L^{q}\left(\left[0, t_{*}\right]\right)} \leq 1$ for some $q>2$ sufficiently close to 2 , an arbitrarily small $\delta>0$ and a sufficiently large integer $m>0$ such that for all $t \in\left[0, t_{*}\right]$ and the projection $P$ on the frequencies of size 1 ,

$$
\|P \partial \psi(t)\|_{L_{x}^{\infty}} \leq C\left(B_{0}\right)\left(\frac{1}{\left(1+\left|t-t_{0}\right|\right)^{1-\delta}}+d(t)\right) \sum_{k=0}^{m}\left\|\nabla^{k} \partial \psi\left(t_{0}\right)\right\|_{L_{x}^{2}} .
$$

This reduction finishes the first part of the proof of the Main Theorem.

The proof of theorem 2.1 crucially relies on the information provided by the Geometric Theorem. The bootstrap conditions (18) guarantee ${ }^{7}$ the assumptions of the Geometric Theorem for the metric $\mathrm{g}$. In fact, we make use of the version of this theorem stated in the Remark 1.5 for the rescaled metric $H$. This means that the size of the allowed neighborhood is $\lambda^{1-\epsilon} C\left(B_{0}\right)$ and the estimates (10), (11) should be adjusted for the rescaling.

\section{Generalized energy estimates and the vectorfield method [Kl-Ro1]}

For the second part of the proof of the Main Theorem we assume the Geometric Theorem, postponing its proof, and establish the decay estimate of Theorem 2.1. Its proof relies on a purely geometric argument based on the construction of a modified Morawetz vectorfield $K$. We construct it with the help of the optical function $u$ associated with the metric $H$.

The modified Morawetz vectorfield is defined according to the formula

$$
K=\underline{u}^{2} L+u^{2} \underline{L}, \quad \underline{u}=2 t-u
$$

with the null frame $(L, \underline{L})$ defined as in (7) relative to the metric $H$. The standard generalized energy identity associated to $K$ takes the form

$$
\int_{\Sigma_{t}} \bar{Q}(K, T)=\int_{\Sigma_{t_{0}}} \bar{Q}(K, T)-\frac{1}{2} \int_{\left[t_{0}, t\right] \times \mathbb{R}^{3}} Q^{\alpha \beta(K)} \bar{\pi}_{\alpha \beta}+\int_{\left[t_{0}, t\right] \times \mathbb{R}^{3}} \psi^{2} \square_{H}(t)
$$

where $Q$ and $\bar{Q}$ are the standard and the modified energy momentum tensors, see [Kl1], [Kl-Ro1], or [Kl-Ro], associated to the equation $\square_{H} \psi=0$. Also,

$$
{ }^{(K)} \bar{\pi}_{\mu \nu}=\mathcal{L}_{K} H_{\mu \nu}-4 t H_{\mu \nu}=D_{\mu} K_{\nu}+D_{\nu} K_{\mu}-4 t H_{\mu \nu}
$$

\footnotetext{
${ }^{7}$ modulo the characteristic energy norm $\left\|D_{*} \mathbf{g}\right\|_{L_{u}^{\infty} H^{s-1}\left(C_{u}\right)}$ which can be also shown to be controlled by the bootstrap constant $B_{0}$
} 
is the modified deformation tensor of the vectorfield $K$.

We show that the decay estimate (22) follows from the the boundedness theorem:

$$
\int_{\Sigma_{t}} \bar{Q}(K, T) \lesssim \int_{\Sigma_{t_{0}}} \bar{Q}(K, T) .
$$

This requires the control of the error term on the right hand side of (23). For this we need good estimates for ${ }^{(K)} \bar{\pi}$ which depend on the control of the geometric quantities $\chi, \eta$. The desired control is given precisely by the estimates (10) and (11) of the Geometric Theorem.

\section{Proof of the Geometric Theorem [K1-Ro2]}

It finally remains to establish the Geometric Theorem. The proof once again relies on the bootstrap argument in which we assume the existence of the foliations $C_{u}$ and $S_{t, u}$ as well the desired estimates (10), (11). We then reestablish the bounds with improved values of the constants and show that the estimates hold in the region whose size only depends on the $\mid\|\mathbf{g}\| \|_{s}$ for some $s>2$. The quantities $\operatorname{tr} \chi, \hat{\chi}$, and $\eta$ satisfy $^{8}$ the null structure equations, which can be written approximately in the form

$$
\begin{aligned}
& L(\operatorname{tr} \chi)+\frac{1}{2}(\operatorname{tr} \chi)^{2}=-|\hat{\chi}|^{2}, \\
& (\text { dijv } \hat{\chi})_{A}=\frac{1}{2} \underset{A}{\not} \operatorname{tr} \chi-\mathbf{R}_{B L A B}, \\
& \text { dijv } \eta=\frac{1}{2} \mu-\frac{1}{2} \mathbf{R}_{B L \underline{L} B}, \quad \text { cuprl } \eta=-\frac{1}{2} \in{ }^{A B} \mathbf{R}_{B L \underline{L} A} .
\end{aligned}
$$

Here $\not$, di/v , and curl are the covariant derivative, divergence, and curl induced on the 2-surfaces $S_{t, u}$. Equation (24) is the transport equation along the integral curves $^{9}$ of the vectorfield $L$. It in fact follows from the Jacobi equation for the Hessian of the optical function $u$ and is also known as the Raychaudhuri equation. The equations (25) and (26) are the elliptic Codazzi equations for $\hat{\chi}$ and $\eta$ on the 2 -surfaces $S_{t, u}$. The quantity $\mu$ is related to the so called mass aspect function and also satisfies a transport equation.

The null structure equations for the null components of the Hessian of $u$ played an important role in the proof of stability of Minkowski space of [Ch-Kl], and in the context of a double foliation in [Kl-Ni].

Integrating the transport equation (24) we can show that approximately

$$
\left|\operatorname{tr} \chi-\frac{2}{n(t-u)}\right| \leq C \int_{\gamma}|\hat{\chi}|^{2}
$$

where $\gamma$ is the integral curve of $L$ connecting a given point with the time axis $\Gamma_{t}$. Thus using the bootstrap assumption on the $\|\hat{\chi}\|_{L_{t}^{2} L_{x}^{\infty}}$ we can reestablish an $L_{t}^{2} L_{x}^{\infty}$

${ }^{8}$ The estimates (10) for $k$ follow immediately from the bootstrap assumption for $\mathbf{g}$ and (8)

${ }^{9}$ In Minkowski space these are the lines $t=\omega \cdot x$ for any $\omega \in S^{2}$ 
estimate for $\operatorname{tr} \chi-\frac{2}{n(t-u)}$. The equation (25) is a first order elliptic equation for $\hat{\chi}$ on $S_{t, u}$ and thus the elliptic estimates lead us to the bound ${ }^{10}$

$$
\|\hat{\chi}\|_{L^{\infty}\left(S_{t, u}\right)} \leq C\left\|\operatorname{tr} \chi-\frac{2}{n(t-u)}\right\|_{L^{\infty}\left(S_{t, u}\right)}+\left\|\left.|\not|\right|^{-1} \mathbf{R}_{B L A B}\right\|_{L^{\infty}\left(S_{t, u}\right)}
$$

The crucial observation is that the $\mathbf{R}_{B L A B}$ component of curvature can be decomposed as follows

$$
\mathbf{R}_{B L A B}=\not \pi+E,
$$

where $|\pi| \leq C|\partial \mathbf{g}|$ and $|E| \leq C|\partial \mathbf{g}|^{2}$. This allows us to recover the $L_{t}^{2} L_{x}^{\infty}$ estimate for $\hat{\chi}$ in terms of the \|\| $\mathbf{g} \| \mid$. Finally, equations (26) form an elliptic system for $\eta$. Once again, the curvature components appearing on the right hand side of these equations decompose in a way similar to (27). Thus arguing as in the case of the estimate for $\hat{\chi}$ we can obtain the $L_{t}^{2} L_{x}^{\infty}$ estimate for $\eta$. The only subtle difference is the control of the quantity $|\not \nabla|^{-1} \mu$. Here we can show that this term is related to $\operatorname{tr} \chi$ and satisfies a transport equation which, crucially, has only mild dependence on the curvature. This is where we heavily use the fact that the metric $\mathbf{g}$ is Einstein.

The estimates (11) can be obtained along the same lines. There the important role is played by the characteristic energy part of the triple norm of $\mathbf{g}$.

Since we actually prove Geometric Theorem for the metric $\mathbf{g}_{<\lambda}=P_{<\lambda} \mathbf{g}$ which is obtained from the Einstein metric $\mathbf{g}$ by truncating the frequencies above $\lambda$, we cannot assume that its Ricci curvature vanishes. This introduces additional terms in the null structure equations. We call the Ricci curvature of a metric, obtained by a frequency truncation from an Einstein metric, the Ricci defect. In [Kl-Ro3] we discover a cancellation property for the $\mathbf{R}_{L L}$ component of the Ricci defect. This is important for the control of the analysis of the null structure equations for a frequency truncated metric.

\section{References}

[An-Mo] L. Andersson and V. Moncrief Elliptic-hyperbolic systems and the Einstein equations. preprint

[Ba-Ch1] H. Bahouri and J. Y. Chemin. Équations d'ondes quasilinéaires et estimation de Strichartz. Amer. J. Math., vol. 121; (1999), pp. 1337-1777

[Ba-Ch2] H. Bahouri and J. Y. Chemin. Équations d'ondes quasilinéaires et effet dispersif. IMRN, vol. 21; (1999), pp. 1141-1178

[Br] Y. Choquet Bruhat. Théorème d'éxistence pour certains systèmes d'équations aux dérivées partielles nonlinéaires., Acta Math. 88 (1952), 141-225.

\footnotetext{
${ }^{10}$ To actually obtain the $L^{\infty}$ bound we need a slightly more regular norm of the right hand side. In [Kl-Ro2] we do this by showing first that we have a "weak" (with a loss of powers of $\lambda$ ) bound for the $\not$ derivative of $\hat{\chi}$. This is the main reason for the frequency truncation of the metric noted in Remark 1.5. Otherwise the norm $\|\not \hat{\chi}\|_{L^{\infty}}$ would be simply infinite. However a more precise argument should be able to allow us to bypass the truncation requirement
} 
[Ch-Kl] D.Christodoulou and S. Klainerman. The Global Nonlinear Stability of the Minkowski Space. Princeton Mathematical Series, 41. Princeton University Press, 1993

[Ha-El] S. Hawking and G. Ellis. The large scale structure of spacetime. Cambridge Monographs on Mathematical Physics, 1973

[H-K-M] Hughes, T. Kato and J. Marsden. Well posed quasilinear second order hyperbolic systems Arch. Rat. Mech. Anal. 63(1976) no 3, 273-294.

[Kl1] S. Klainerman. A commuting vectorfield approach to Strichartz type inequalities and applications to quasilinear wave equations. IMRN, 2001, No 5, 221-274.

[Kl2] S. Klainerman. PDE as a unified subject Special Volume GAFA 2000, 279-315

[Kl-Ni] S. Klainerman and F. Nicolo. On the initial value problem in General Relativity. preprint

[Kl-Ro] S. Klainerman and I. Rodnianski. Improved local well posedness for quasilinear wave equations in dimension three. to appear in Duke Math. Journ.

[Kl-Ro1] S. Klainerman and I. Rodnianski. Rough solutions of the Einstein-vacuum equations . preprint

[Kl-Ro2] S. Klainerman and I. Rodnianski. The causal structure of microlocalized, rough, Einstein metrics . preprint

[Kl-Ro3] S. Klainerman and I. Rodnianski. Ricci defects of microlocalized, rough, Einstein metrics .preprint

[Li] H. Lindblad, Counterexamples to local existence for semilinear wave equations. AJM, vol. 118; (1996), pp. 1-16

[Po-Si] G. Ponce and T. Sideris. Local regularity of non linear wave equations in three space dimensions. CPDE, vol. 18; (1993), pp. 169-177

[Sm] H. Smith. A parametrix construction for wave equations with $C^{1,1}$ coeffcients. Annales de L'Institut Fourier, vol. 48; (1998), pp. 797-835

[Sm-So] H. Smith and C. Sogge. On Strichartz and eigenfunction estimates for low regularity metrics. Math. Res. Lett., vol. 1; (1994), pp. 729-737

[Sm-Ta1] H. Smith and D. Tataru. Sharp counterexamples for Strichartz estimates for low regularity metrics. Preprint

[Sm-Ta2] H. Smith and D. Tataru. Sharp local well-posedness results for the nonlinear wave equation. Preprint

[Ta2] D. Tataru. Strichartz estimates for second order hyperbolic operators with non smooth coefficients. Preprint 
[Ta1] D. Tataru. Strichartz estimates for operators with non smooth coefficients and the nonlinear wave equation. Amer. J. Math., vol. 122; (2000), pp. 349-376

Department of Mathematics, Princeton University, PRINCETON NJ 08544

seri@@math.princeton. edu

irod@@math.princeton. edu 